\title{
Treponemal Infection
}

National Cancer Institute

\section{Source}

National Cancer Institute. Treponemal Infection. NCI Thesaurus. Code C85197.

A sexually transmitted infection caused by the Gram-negative spirochete Treponema pallidum. It is manifested as syphilis. 